\title{
In vivo Assessment of Combined Effects of Glibenclamide and Losartan in Diabetic Rats
}

\author{
Moureq R. Alotaibi ${ }^{a} \quad$ Amal J. Fatani ${ }^{a} \quad$ Ahmed T. Almnaizel $^{a}$ \\ Mohammed M. Ahmed ${ }^{a}$ Hatem M. Abuohashish $^{b}$ Salim S. Al-Rejaie ${ }^{a}$ \\ a Department of Pharmacology and Toxicology, College of Pharmacy, King Saud University, Riyadh, Saudi Arabia; \\ ${ }^{b}$ Department of Biomedical Dental Sciences, College of Dentistry, Imam Abdulrahman Bin Faisal University, \\ Dammam, Saudi Arabia
}

\section{Significance of the Study}

- A combination of glibenclamide and losartan therapy diminished the consequences of diabetic hyperglycemia in this study. The combined therapy showed a benefit on hyperglycemia-induced oxidative and inflammatory reactions. However, the combined effects did not demonstrate a significant advantage over single drug therapy. These findings may be of relevance to the treatment of hypertensive diabetics.

\section{Keywords}

Diabetes mellitus · Glibenclamide · Losartan · Combined therapy · Oxidative stress · Inflammation

\begin{abstract}
Objective: Diabetic complications involve multiple pathological pathways, including hyperglycemia-induced oxidative stress and inflammation. Combination therapy is usually employed to improve treatment outcomes and to lower potential adverse effects. In this study, we evaluated the effects of antidiabetic and antihypertensive agents, glibenclamide (GLI) and losartan (LT), on diabetes mellitus (DM)associated metabolic changes in rats. Materials and Methods: Streptozotocin-induced diabetic animals were orally treated with GLI 5 mg/kg and/or LT 25 mg/kg for 4 weeks.
\end{abstract}

Blood glucose, insulin, aspartate aminotransferase, alanine aminotransferase, urinary creatinine, and urea levels were measured. Serum, liver, and kidney values of inflammatory markers, such as interleukin-1 $\beta$, tumor necrosis factor alpha, and interleukin- 6 were assessed, along with lipid peroxidation products (e.g., thiobarbituric acid reactive substances), endogenous antioxidants (e.g., glutathione), as well as antioxidant enzyme activities (e.g., catalase, superoxide dismutase, and glutathione peroxidase). Finally, histological changes in liver and kidney tissues were evaluated. Results: DM markedly induced systemic, hepatic, and renal inflammation and lowered antioxidant defense mechanisms. Treatment of diabetic rats with either GLI or LT significantly improved liver and kidney functions and histological structure. Moreover, both medications reduced signs of oxidative stress and inflammation in blood, liver, and kidney sam-

\begin{tabular}{ll}
\hline KARGER & $\begin{array}{l}\text { ( 2018 The Author(s) Karger } \\
\text { Published by S. Karger AG, Basel Open cacess }\end{array}$ \\
E-Mail karger@karger.com & $\begin{array}{l}\text { This is an Open Access article licensed under the Creative Commons } \\
\text { Attribution-NonCommercial-4.0 International License (CC BY-NC) } \\
\text { (http://www.karger.com/Services/OpenAccessLicense), applicable to } \\
\text { the online version of the article only. Usage and distribution for } \\
\text { commercial purposes requires written permission. }\end{array}$
\end{tabular}

Salim S. Al-Rejaie

Department of Pharmacology and Toxicology

College of Pharmacy, King Saud University

P.O. Box 55760, Riyadh 11544 (Saudi Arabia)

E-Mail rejaie@ksu.edu.sa or rejaie@ hotmail.com 
ples. Combining GLI and LT showed similar protective potential against systemic, hepatic, and renal oxidative stress and inflammation. Conclusion: Adding LT to GLI therapy revealed prospective antioxidant and anti-inflammatory action, while no synergistic or additive effects were observed.

(c) 2018 The Author(s)

Published by S. Karger AG, Basel

\section{Introduction}

Diabetes mellitus (DM) is an endocrine disorder characterized by multiple etiological factors, including chronic hyperglycemia and alterations in the metabolism of carbohydrates, fats, and proteins caused by defects in insulin secretion and/or action. The associated chronic hyperglycemia may lead to long-term oxidative cellular injury and dysfunction of various organs, particularly the kidneys, eyes, nerves, liver, heart, and blood vessels. The provoked oxidative stress and lipid peroxidation act as a potential pathophysiological mechanism underlying diabetic complications through the overproduction of reactive oxygen species and by triggering the production of proinflammatory cytokines, which together with reactive oxygen species are deemed to play vital roles in the pathogenesis of DM [1]. Furthermore, the use of antioxidants has been found to reduce the burden of diabetic complications in several experimental and clinical studies [1-3].

Glibenclamide (GLI) is a common oral hypoglycemic agent that works primarily by stimulating insulin secretion via blockage of the ATP-sensitive $\mathrm{K}^{+}$channels and depolarization of pancreatic beta cells. GLI also functions by the same mechanism of action in extrapancreatic sites, including the liver, kidneys, heart, as well as skeletal and smooth muscles [4]. In addition, the ability of GLI to reduce the biomarkers of oxidative stress and lipid peroxidation during DM has been documented in several experimental studies where GLI was used as a reference antidiabetic agent [5].

The renin-angiotensin system (RAS) is a well-documented endocrine system that regulates the physiological electrolyte and fluid balance. The octapeptide angiotensin-II induces vasoconstriction and proinflammatory markers. Inhibition of the production or action of angiotensin-II is a target for different groups of antihypertensive agents, including angiotensin-converting enzyme inhibitors and angiotensin-II type $1\left(\mathrm{AT}_{1}\right)$ receptor blockers. Evidence supports the pathological role of RAS components in the development and progression of diabetic complications by exacerbating oxidative injury and inflammation [6]. Losartan (LT) is an $\mathrm{AT}_{1}$ receptor block- er that is characterized by its antioxidant defense properties during DM [7]. Moreover, LT reduced DM-induced inflammation and lipid peroxidation in diabetic rats [8].

In recent decades, there has been increasing interest in combination therapy or polytherapy, which refers to the concurrent consumption of multiple medications against one or several medical conditions. As hypertension is considered one of the macrovascular complications of $\mathrm{DM}$, the concomitant use of GLI and LT in diabetic patients could be beneficial to treating DM. Therefore, this study was designed to evaluate the impact of combined GLI and LT therapies on oxidative and inflammatory changes in diabetic rats.

\section{Materials and Methods}

\section{Animals}

Male Wistar albino rats weighing between 260 and $300 \mathrm{~g}$ were obtained from the Experimental Animal Care Center, College of Pharmacy, King Saud University.

\section{Experimental Procedures}

DM was induced by a single intraperitoneal injection of $60 \mathrm{mg} /$ $\mathrm{kg}$ streptozotocin (STZ). STZ was freshly dissolved in citrate buffer $(0.1 \mathrm{M}, \mathrm{pH}=4.5)$. The remaining control animals were injected with an equivalent volume of citrate buffer. After $72 \mathrm{~h}$, blood glucose levels were measured with a glucometer (ACCU-CHEK Active, Mannheim, Germany), and values $>250 \mathrm{mg} / \mathrm{dL}$ were considered diabetic. Animals were then randomly divided into five groups ( $n=6$ each) as follows: (A) control group; (B) DM (STZ) group; (C) DM + GLI group (5 mg/kg oral dose); (D) DM + LT group (25 mg/kg oral dose); and (E) DM + GLI + LT group (5 and $25 \mathrm{mg} / \mathrm{kg}$ oral dose, respectively). GLI (Sigma-Aldrich Chemie, Steinheim, Germany) and LT (TRC Inc., Toronto, ON, Canada) were suspended in $0.5 \%$ carboxymethyl cellulose and administered via gastric gavage in a volume of $0.5 \mathrm{~mL} / 100 \mathrm{~g}$ body weight. The doses for GLI and LT were selected in line with previous experimental reports $[9,10]$. The control and STZ groups received similar volumes of $0.5 \%$ carboxymethyl cellulose during the treatment period. Treatment started 1 week after the induction of DM and lasted for 4 consecutive weeks, which is sufficient time for diabetic metabolic changes to appear. Then 24 -h urine samples were collected in metabolic cages, and after centrifugation at 3,000 rpm for $5 \mathrm{~min}$, the supernatants were transferred to $1.5-\mathrm{mL}$ tubes. Next, blood samples were collected via cardiac puncture and centrifuged at 3,000 rpm for $10 \mathrm{~min}$ to obtain the sera. Liver and kidney tissues were desiccated, weighed, and homogenized in a 10-fold volume of ice-cold phosphate-buffered saline $(\mathrm{pH}=7.4)$. The homogenates were transferred to $1.5-\mathrm{mL}$ tubes and stored at $-80^{\circ} \mathrm{C}$ until use.

\section{Biochemical Analysis}

Serum glucose and insulin levels were measured by a commercially available kit (RANDOX Laboratories Ltd., UK and SPI bio, France, respectively). Serum aspartate aminotransferase and alanine aminotransferase were determined by diagnostic kits $(\mathrm{Hu}-$ 
Table 1. Effects of GLI and/or LT on serum and urine biochemistry in diabetic rats

\begin{tabular}{|c|c|c|c|c|c|}
\hline & Control & STZ & GLI & LT & $\mathrm{GLI}+\mathrm{LT}$ \\
\hline Glucose, $\mathrm{mmol} / \mathrm{L}$ & $4.8 \pm 1.56$ & $11.2 \pm 1.45^{\mathrm{a}}$ & $6.1 \pm 0.79^{\mathrm{b}}$ & $9.8 \pm 1.05^{\mathrm{a}}$ & $6.4 \pm 1.37^{\mathrm{b}}$ \\
\hline Insulin, mAU & $476.5 \pm 41.38$ & $138.9 \pm 21.77^{\mathrm{a}}$ & $290.3 \pm 20.16^{b}$ & $143.3 \pm 13.73^{\mathrm{a}}$ & $196.3 \pm 28.42^{\mathrm{b}}$ \\
\hline TNF- $\alpha, p g / m L$ & $85.1 \pm 4.64$ & $126.5 \pm 12.49^{\mathrm{a}}$ & $106.3 \pm 10.41^{b}$ & $113.1 \pm 6.08^{\mathrm{b}}$ & $116.4 \pm 8.83^{\mathrm{b}}$ \\
\hline $\mathrm{IL}-6, \mathrm{pg} / \mathrm{mL}$ & $197.5 \pm 12.28$ & $353.6 \pm 129.12^{\mathrm{a}}$ & $247.3 \pm 11.92^{b}$ & $221.8 \pm 18.31^{b}$ & $228.6 \pm 15.65^{b}$ \\
\hline TBARS, nmol/mL & $299.7 \pm 35.41$ & $546.5 \pm 33.91^{\mathrm{a}}$ & $423 \pm 20.57^{b}$ & $350.5 \pm 59.27^{\mathrm{b}}$ & $370.7 \pm 42.41^{b}$ \\
\hline $\mathrm{CAT}, \mathrm{nmol} / \mathrm{min} / \mathrm{mL}$ & $8.5 \pm 0.32$ & $3.8 \pm 0.25^{\mathrm{a}}$ & $9.8 \pm 0.75^{\mathrm{b}}$ & $9 \pm 0.21^{b}$ & $7.6 \pm 0.24^{\mathrm{b}}$ \\
\hline SOD, U & $25.5 \pm 1.36$ & $17.2 \pm 1.93^{\mathrm{a}}$ & $23.5 \pm 0.65^{\mathrm{b}}$ & $22.4 \pm 1.57^{\mathrm{b}}$ & $23.3 \pm 0.72^{\mathrm{b}}$ \\
\hline $\mathrm{GPx}, \mathrm{mU} / \mathrm{L}$ & $7.7 \pm 0.81$ & $5.3 \pm 0.26^{\mathrm{a}}$ & $6.8 \pm 0.34^{\mathrm{b}}$ & $8.7 \pm 0.42^{\mathrm{b}}$ & $8.4 \pm 0.42^{\mathrm{b}}$ \\
\hline AST, U/L & $37.1 \pm 2.75$ & $49.7 \pm 2.35^{\mathrm{a}}$ & $38.7 \pm 2.57^{\mathrm{b}}$ & $39.9 \pm 1.77^{\mathrm{b}}$ & $40.8 \pm 2.55^{\mathrm{b}}$ \\
\hline ALT, U/L & $30.5 \pm 1.48$ & $105.5 \pm 2.93^{\mathrm{a}}$ & $89.9 \pm 1.46^{\mathrm{b}}$ & $81.5 \pm 3.53^{b}$ & $97.3 \pm 2.51^{\mathrm{b}}$ \\
\hline Urinary creatinine, $\mathrm{mmol} / \mathrm{L}$ & $8.3 \pm 2.25$ & $3.5 \pm 0.54^{\mathrm{a}}$ & $5.7 \pm 0.77^{\mathrm{b}}$ & $5 \pm 0.72^{\mathrm{b}}$ & $5.9 \pm 0.52^{\mathrm{b}}$ \\
\hline
\end{tabular}

Numerical values are presented as mean \pm standard deviation and were statistically analyzed using one-way ANOVA followed by Newman-Keuls multiple comparison test. ALT, alanine aminotransferase; AST, aspartate aminotransferase; CAT, catalase; GLI, glibenclamide; GPx, glutathione peroxidase; GSH, glutathione; IL-1 $\beta$, interleukin-1 $\beta$; IL-6, interleukin-6; LT, losartan; SOD, superoxide dismutase; STZ, streptozotocin; TBARS, thiobarbituric acid reactive substances; TNF- $\alpha$, tumor necrosis factor alpha. Statistically significant difference: ${ }^{\mathrm{a}} p<0.01$ versus control group, ${ }^{\mathrm{b}} p<0.01$ versus STZ group.

man Diagnostics Worldwide, Wiesbaden, Germany). Urine creatinine and urea were estimated by colorimetric methods (Linear Chemicals, Barcelona, Spain). Serum, liver, and kidney levels of interleukin-1 $\beta$ (IL-1 $\beta$ ), tumor necrosis factor alpha (TNF- $\alpha$ ), and interleukin-6 (IL-6) were determined by an ELISA technique (Thermo Scientific, Rockford, IL, USA).

Estimation of Lipid Peroxidation and Oxidative Stress Markers

Serum, liver, and kidney concentrations of thiobarbituric acid reactive substances (TBARS) were assayed by a biochemical kit provided by Zepto Metrix Inc., USA. Levels of reduced glutathione (GSH) were determined by the Sedlak and Lindsay method [11]. The enzymatic activities of catalase (CAT), superoxide dismutase (SOD), and glutathione peroxidase (GPx) were evaluated by kits (Cyman Chemical Company, Ann Arbor, MI, USA).

\section{Histology}

Liver and kidney specimens were fixed in 10\% formalin and subsequently sliced into segments and embedded in paraffin wax squares. Then the paraffin pieces were cut with a microtome and stained with hematoxylin and eosin. The recolored segments were mounted and examined for histopathological variations by a histopathologist in a blinded manner. Histological assessment of liver injury was conducted as follows. Grading of hepatocyte architecture: (0) no fibrosis, (1) $\leq 25 \%$ fibrotic damage, (2) $26-50 \%$ fibrotic damage, (3) 51-75\% fibrotic damage, and (4) $>75 \%$ fibrotic damage. Grading of inflammatory infiltrate: (0) no lymphocytes, (1) $\leq 25 \%$ of lymphocytes and inflammatory exudate, (2) $26-50 \%$ of lymphocytes and inflammatory exudate, (3) 51-75\% of lymphocytes and inflammatory exudate, and (4) $>75 \%$ of lymphocytes and inflammatory exudate. Histological grading of kidney injury:
Grading of glomerular damage: (0) no damage, (1) $\leq 25 \%$ of glomeruli involved, (2) $26-50 \%$ of glomeruli involved, (3) $51-75 \%$ of glomeruli involved, and (4) $>75 \%$ of glomeruli involved. Grading of acute tubular necrosis: ( 0 ) no damage, $(1) \leq 25 \%$ of renal tubules involved, (2) $26-50 \%$ of renal tubules involved, (3) $51-75 \%$ of renal tubules involved, and (4) $>75 \%$ of renal tubules involved. Grading of tubulointerstitial nephritis: (0) no damage, (1) leukocytes confined within the interstitium, and (2) leukocytes within the interstitium and tubular epithelial cells.

\section{Statistical Analysis}

The results of the current study are presented as the mean \pm standard deviation. Statistical analyses were carried out using oneway ANOVA followed by a Newman-Keuls multiple comparison test applied on GraphPad Prism version 5. Differences were considered statistically significant when $p$ values were $<0.05$.

\section{Results}

Glucose, aspartate aminotransferase, and alanine aminotransferase levels increased significantly $(p<0.01)$ in the STZ group compared to the control group, while these levels were significantly decreased in the GLI- and LTtreated groups compared to STZ $(p<0.01)$ (Table 1$)$. The insulin concentration in the STZ group showed significantly lower levels relative to those of the control group $(p<0.01)$, while insulin levels significantly increased in 
the GLI group $(p<0.01)$ compared to the STZ group. The diabetic groups that were treated showed a significant increase in urine creatinine in comparison with the STZ controls $(p<0.01)$. On other hand, there were significant decreases in urine urea levels in the treated groups compared to those in the STZ group $(p<0.01)$ (Table 1).

The serum, liver, and kidney levels of IL- $1 \beta$, TNF- $\alpha$, and IL-6 showed a significant $(p<0.01)$ increase in the STZ group compared to the control group. All treated groups demonstrated significant $(p<0.01)$ corrected values of IL-1 $\beta$, TNF- $\alpha$, and IL-6 (Tables 1,2 ). In the STZ group serum, hepatic, and renal levels of TBARS were significantly elevated $(p<0.01)$, while GSH levels were significantly reduced $(p<0.01)$ compared to control animals. Treatment of the animals with GLI and/or LT significantly $(p<0.01)$ attenuated the altered TBARS and GSH concentrations (Tables 1,2). The enzymatic activities of CAT, SOD, and GPx enzymes were significant $(p<0.01)$ in the diabetic untreated group compared to the control group. The altered antioxidant enzymatic activities were significantly $(p<0.01)$ corrected in the GLI, LT, and GLI + LT groups compared to the STZ group (Tables 1, 2).

Histopathological assessment of liver sections revealed normal- and benign-looking liver hepatocytes in the control animals (Fig. 1a). In the diabetic untreated rats the hepatocytes were degenerated, dilated, and surrounded by inflammatory cells infiltrating along with fatty degeneration (Fig. 1b). Treatment of diabetic animals with GLI reduced hepatocellular fibrosis, degeneration, and inflammation (Fig. 1c). Hepatocytes from the diabetic group treated with LT looked benign and well-arranged in structure, with limited inflammatory exudates (Fig. 1d). Similarly, liver sections from the diabetic group treated with GLI and LT in combination showed normal and well-structured hepatocytes without fibrosis or degenerative changes (Fig. 1e).

Histopathological evaluation of kidney sections revealed normal- and benign-looking renal tissues along with prominent cortex and medulla in the control animals (Fig. 2a). In the diabetic untreated group, there were significantly congested renal tubules with scattered intraluminal eosinophilic sections (Fig. 2b). Diabetic animals treated with GLI had preserved renal structure with a few scattered eosinophilic intratubular secretions (Fig. 2c). LT markedly preserved the renal cortex and medulla and reduced the cloudy degeneration of renal tubules in the kidneys of diabetic animals (Fig. 2d). Kidney sections from diabetic animals treated with GLI and LT in combination had perfect and normal appearance

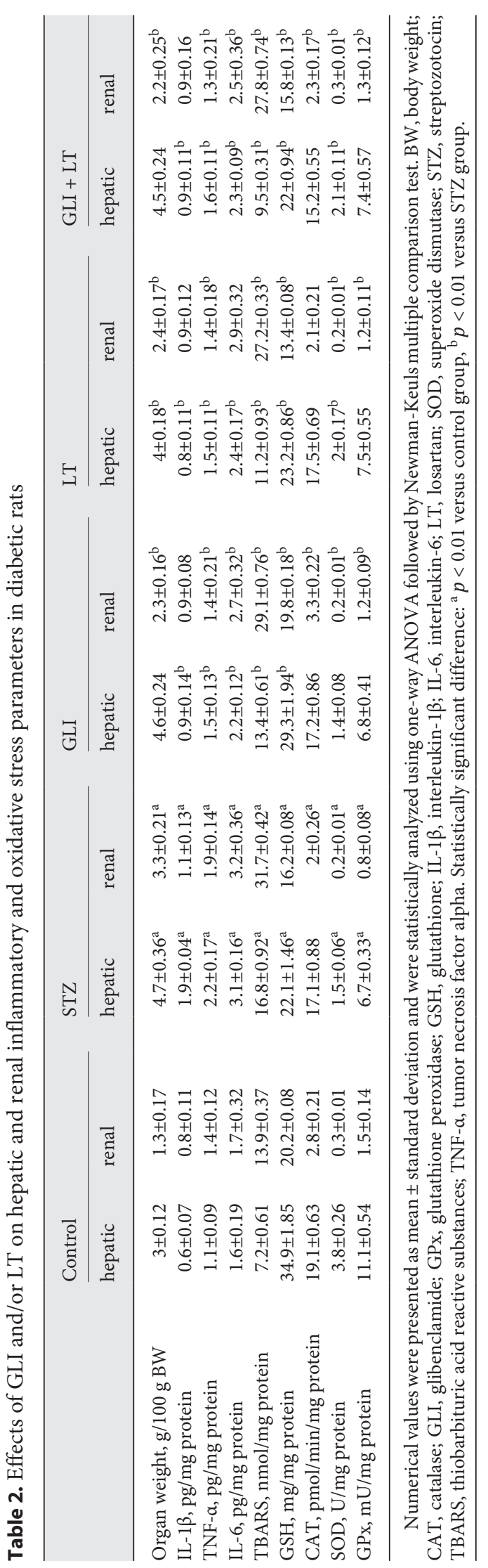




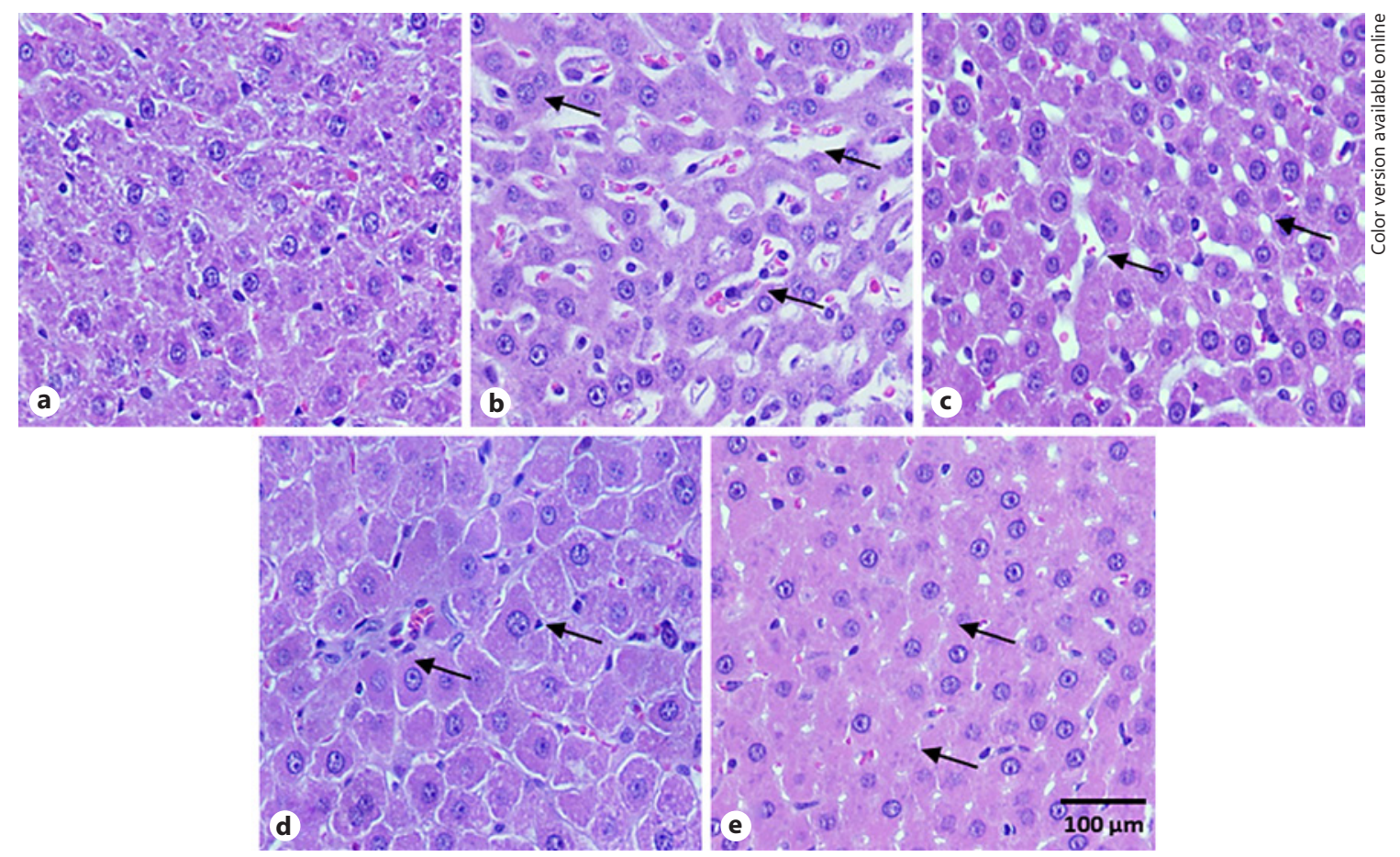

Fig. 1. Effects of GLI and/or LT on liver histopathological changes in diabetic rats $(\times 400)$. a Liver of a control animal showing benign normal-looking hepatocytes. b Liver of a diabetic untreated animal. The arrows indicate inflammatory cell infiltrate with ballooning degeneration and few fatty degenerated cells. c Liver of a diabetic animal treated with GLI. The arrows show limited peripor- tal inflammatory cells with no fibrosis and degenerative changes. d Liver of a diabetic animal treated with LT. The arrows demonstrate benign well-arranged hepatocytes with few inflammatory infiltrate. e Liver of a diabetic animal treated with GLI and LT. The arrows indicate typical-looking hepatocytes with no degenerated cells or inflammatory infiltrate. GLI, glibenclamide; LT, losartan.

Table 3. Histological assessment of liver and kidney injuries in diabetic rats

\begin{tabular}{|c|c|c|c|c|c|}
\hline & Control & STZ & GLI & LT & $\mathrm{GLI}+\mathrm{LT}$ \\
\hline \multicolumn{6}{|l|}{ Liver } \\
\hline Hepatocyte architecture & 0 & 3 & 2 & 1 & 1 \\
\hline Inflammatory infiltrate & 0 & 3 & 2 & 2 & 1 \\
\hline Total score & 0 & 6 & 4 & 3 & 2 \\
\hline Liver injury grade & no & moderate & mild & mild & mild \\
\hline \multicolumn{6}{|l|}{ Kidneys } \\
\hline Glomerular damage & 0 & 3 & 1 & 2 & 1 \\
\hline Acute tubular necrosis & 0 & 2 & 2 & 1 & 2 \\
\hline Tubulointerstitial nephritis & 0 & 2 & 1 & 1 & 1 \\
\hline Total score & 0 & 7 & 4 & 4 & 4 \\
\hline Kidney injury grade & no & moderate & mild & mild & mild \\
\hline
\end{tabular}

GLI, glibenclamide; LT, losartan; STZ, streptozotocin. The total score of liver samples evaluation indicated the degree for liver injury, where $0-1$ classified as no injury, 2-4 as mild injury, 5-6 as moderate injury, and 7-8 as severe injury. The degree of kidney injury was evaluated based on the renal total score, where $0-1$ classified as no injury, 2-4 as mild injury, 5-7 as moderate injury, and 8-10 as severe injury. 


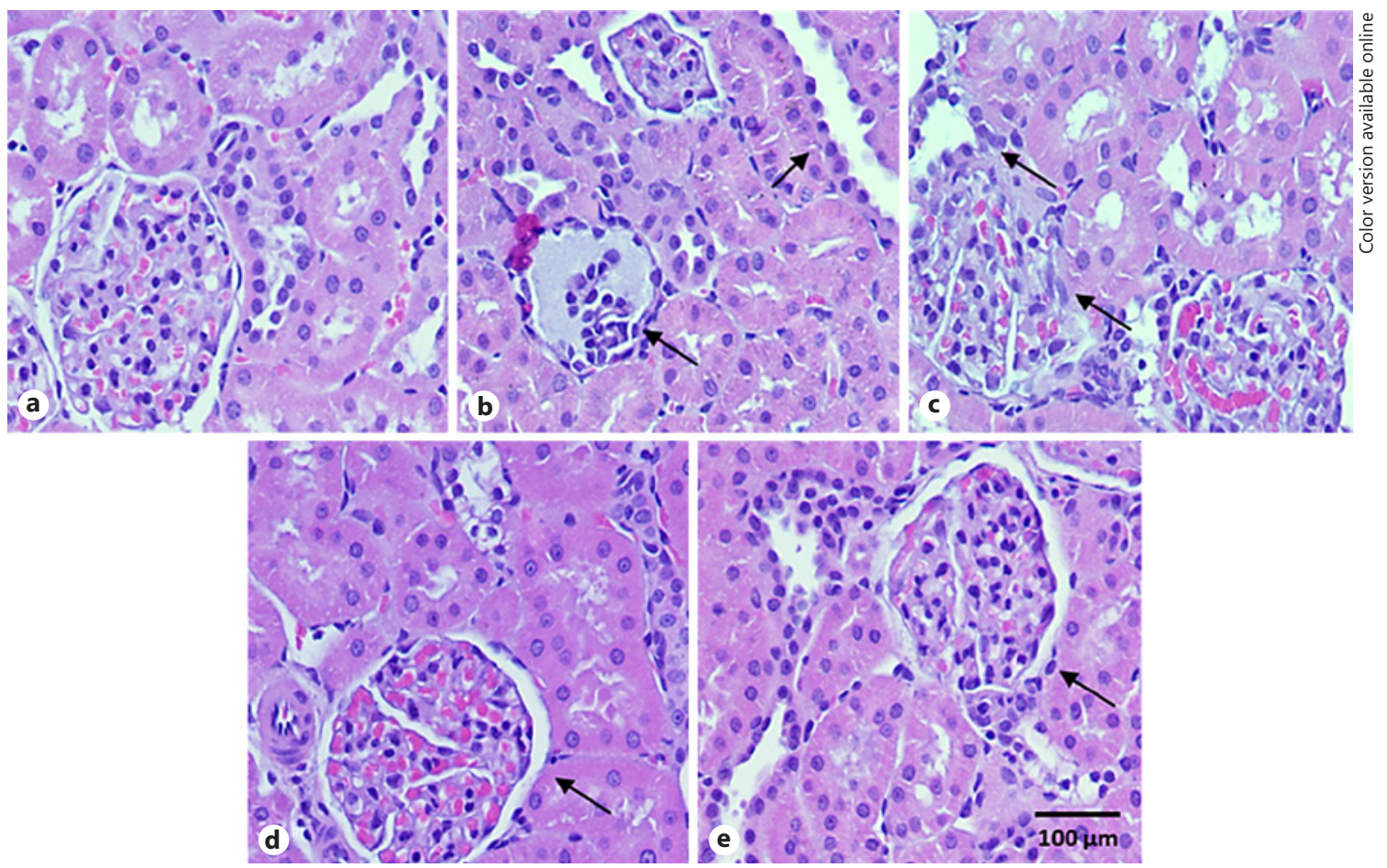

Fig. 2. Effects of GLI and/or LT on kidney histopathological changes in diabetic rats $(\times 400)$. a Kidney of a control animal showing normal benign-looking nephron. $\mathbf{b}$ Kidney of a diabetic untreated animal. The arrows indicate congested and inflamed renal tubules. c Kidney of a diabetic animal treated with GLI. The arrows show preserved renal cortex and medulla with partial inflammation. d Kidney of a diabetic animal treated with LT. The arrow points to conserved renal cortex and medulla with no congestion. e Kidney of a diabetic animal treated with GLI and LT. The arrow points to lack of renal inflammatory exudate or degeneration. GLI, glibenclamide; LT, losartan. of renal glomeruli and tubules (Fig. 2e). The total histological assessment of liver and kidney injuries is provided in Table 3.

\section{Discussion}

Chemically induced DM in rats by STZ is a widely utilized experimental model. STZ accumulates after administration in the beta cells, where glucose transporter type 2 selectively transports it, intracellularly causing DNA alkylation and loss of cellular function [12]. Oxidative stress has been reported in several studies to exacerbate DM complications through triggering lipid and nucleic acid damage along with harming antioxidant defense mechanisms $[13,14]$. Studies have also reported that hyperglycemia provokes the production of proinflammatory cytokines, which contribute to considerable pathology during DM-associated inflammation and apoptosis [15]. Together, oxidative stress and inflammation form a malicious series of biological events that eventually lead to tissue injury. Similarly, signs of augmented systemic and tissue oxidative injuries and inflammation were observed in the present study. Moreover, DM-associated hepatotoxicity was confirmed by elevated liver function enzymes and histological variations, while renal histological analysis and altered creatinine and urea levels verified the renal toxicity in diabetic untreated animals. This was associated with higher levels of proinflammatory cytokines and lipid peroxidation along with diminished endogenous antioxidant molecules and enzyme activities in the STZ group.

In the present study, GLI and LT therapies lowered signs of oxidative stress, lipid peroxidation, and inflammation. Moreover, both medications preserved hepatic and renal functions and structures in diabetic animals. Several clinical and experimental studies have reported comparable protective effects for $\mathrm{AT}_{1}$ receptor antagonism by LT against DM-induced hepatic and renal injuries through its antioxidant and anti-inflammatory prop- 
erties [16]. Interestingly, co-administration of LT with sitagliptin, an antidiabetic agent that belongs to the dipeptidyl peptidase-4 inhibitor group, suppressed the prognosis of nondiabetic nonalcoholic steatohepatitis in diabetic rats [17]. Blockage of the $\mathrm{AT}_{1}$ receptor has been reported to improve liver function in hypertensive patients $[18,19]$. In diabetic rats, LT also provided protection against vascular dysfunction seen in diabetic nephropathy via modulation of renal oxidative stress and inflammation [7]. On the other hand, GLI is a well-known oral antidiabetic agent. Numerous studies have reported its antioxidant and anti-inflammatory properties in different biological systems of diabetic animals [20-22]. Studies have also revealed a marked impact of GLI therapy on DM inflammation and oxidative stress in humans [23]. In contrast, GLI, as a $K_{\text {ATP }}$ channel blocker, abolished sodium hydrosulfide neuroprotection against oxidative stress in one study [24]. Moreover, GLI monotherapy had unfavorable effects on lipid peroxidation in the liver and pancreatic tissues of diabetic animals, suggesting the need for combination therapy to restore the antioxidant potential of the drug [25]. Interestingly, Chatuphonprasert et al. [26] reported that GLI co-therapy with berberine did not provide additional value for either medication, which indicates that the therapeutic value may be restricted to single, but not combined, treatments.

Although both GLI and LT showed significant preventive effects against oxidative stress, inflammation, and histological impairments, we did not observe any synergetic or additive properties. Different sites of action, specifically related to blood glucose lowering abilities, may explain these findings. Furthermore, other studies have reported a conflicting action between GLI and anti-RAS medications [27]. Williams et al. [28] reported that chronic GLI therapy resulted in higher blood pressure following infusion of Ang-II in diabetic patients. In addition,
GLI treatment in dogs markedly inhibited the coronary vasodilatory effect of $\mathrm{AT}_{1}$ receptor blockage, where modulation of $\mathrm{K}_{\mathrm{ATP}}$ channels was deemed to play a vital role [29]. We did not notice a significant change in fasting elevated blood glucose levels in LT-treated diabetic rats either alone or in combination with GLI. Another $\mathrm{AT}_{1}$ receptor antagonist, eprosartan, was also unable to significantly alter the 24 -h plasma glucose profile in patients with type $2 \mathrm{DM}$ who had previously been stabilized on glyburide [30].

In conclusion, the present study revealed the remarkable systemic, hepatic, and renal antioxidant and anti-inflammatory actions of both GLI and LT in diabetic rats. These preventive abilities were neither harmed nor improved by combining both therapies. Therefore, concurrent administration of both medications may have a therapeutic value in diabetic patients without conferring a synergistic benefit.

\section{Acknowledgments}

The authors express their appreciation to the Deanship of Scientific Research at King Saud University for funding the work through Research Group Project No. RGP-VPP-179.

\section{Statement of Ethics}

All experimental protocols were performed in agreement with the Laboratory Animals Usage Guidelines of the National Institutes of Health and ethically approved at the College of Pharmacy, King Saud University.

\section{Disclosure Statement}

The authors declare no conflicts of interest.

\section{References}

1 Abdali D, Samson SE, Grover AK. How effective are antioxidant supplements in obesity and diabetes? Med Princ Pract. 2015;24(3): 201-15.

2 Hasanein P, Riahi H. Antinociceptive and antihyperglycemic effects of Melissa officinalis essential oil in an experimental model of diabetes. Med Princ Pract. 2015;24(1):47-52.

3 Farajpour R, Sadigh-Eteghad S, Ahmadian N, Farzipour M, Mahmoudi J, Majdi A. Chronic Administration of Rosa canina Hydro-Alcoholic Extract Attenuates Depressive-Like Behavior and Recognition Memory Impair- ment in Diabetic Mice: A Possible Role of Oxidative Stress. Med Princ Pract. 2017; 26(3):245-50.

4 Gao R, Yang T, Xu W. Enemies or weapons in hands: investigational anti-diabetic drug glibenclamide and cancer risk. Expert Opin Investig Drugs. 2017 Jul;26(7):853-64.

5 Madhuri K, Naik PR. Ameliorative effect of borneol, a natural bicyclic monoterpene against hyperglycemia, hyperlipidemia and oxidative stress in streptozotocin-induced diabetic Wistar rats. Biomed Pharmacother. 2017 Dec;96:336-47.
6 Kim HJ, Han SJ, Kim DJ, Jang HC, Lim S, Choi SH, et al. Effects of valsartan and amlodipine on oxidative stress in type 2 diabetic patients with hypertension: a randomized, multicenter study. Korean J Intern Med (Korean Assoc Intern Med). 2017 May;32(3): 497-504.

7 Lodovici M, Bigagli E, Tarantini F, Di Serio C, Raimondi L. Losartan reduces oxidative damage to renal DNA and conserves plasma antioxidant capacity in diabetic rats. Exp Biol Med (Maywood). 2015 Nov;240(11): $1500-4$. 
8 Vargas R, Rincón J, Pedreañez A, Viera N, Hernández-Fonseca JP, Peña C, et al. Role of angiotensin II in the brain inflammatory events during experimental diabetes in rats. Brain Res. 2012 May; 1453:64-76.

9 Bolanle IO, Omogbai EK, Bafor EE. Effects of amlodipine and valsartan on glibenclamidetreated streptozotocin-induced diabetic rats. Biomed Pharmacother. 2018 Oct;106:566-74.

10 Liu Y, Zhou D, Xiao X, Qin D, Zhong X, Xiong $\mathrm{X}$, et al. Losartan preserves glomerular basement membrane anionic charge sites in a rat model of nephropathy. J Nephrol. 2013 Jul-Aug;26(4):660-6.

11 Sedlak J, Lindsay RH. Estimation of total, protein-bound, and nonprotein sulfhydryl groups in tissue with Ellman's reagent. Anal Biochem. 1968 Oct;25(1):192-205.

12 Szkudelski T. The mechanism of alloxan and streptozotocin action in B cells of the rat pancreas. Physiol Res. 2001;50(6):537-46.

13 Omidi H, Khorram S, Mesgari M, AsghariJafarabadi M, Tarighat-Esfanjani A. Effects of separate and concurrent supplementation of Nano-sized clinoptilolite and Nigella sativa on oxidative stress, anti-oxidative parameters and body weight in rats with type 2 diabetes. Biomed Pharmacother. 2017 Dec;96:133540.

14 Chang YC, Chuang LM. The role of oxidative stress in the pathogenesis of type 2 diabetes: from molecular mechanism to clinical implication. Am J Transl Res. 2010 Jun;2(3):31631.

15 Adam SH, Giribabu N, Rao PV, Sayem AS, Arya A, Panichayupakaranant $\mathrm{P}$, et al. Rhinacanthin $\mathrm{C}$ ameliorates hyperglycaemia, hyperlipidemia and pancreatic destruction in streptozotocin-nicotinamide induced adult male diabetic rats. Eur J Pharmacol. 2016 Jan; 771:173-90.

16 Kushwaha S, Vikram A, Jena GB. Protective effects of enalapril in streptozotocin-induced diabetic rat: studies of DNA damage, apopto- sis and expression of CCN2 in the heart, kidney and liver. J Appl Toxicol. 2012 Sep;32(9): 662-72.

17 Okura Y, Namisaki T, Moriya K, Kitade M, Takeda K, Kaji K, et al. Combined treatment with dipeptidyl peptidase-4 inhibitor (sitagliptin) and angiotensin-II type 1 receptor blocker (losartan) suppresses progression in a non-diabetic rat model of steatohepatitis. Hepatol Res. 2017 Nov;47(12):1317-28.

18 Hirata T, Tomita K, Kawai T, Yokoyama H, Shimada A, Kikuchi M, et al. Effect of Telmisartan or Losartan for Treatment of Nonalcoholic Fatty Liver Disease: Fatty Liver Protection Trial by Telmisartan or Losartan Study (FANTASY). Int J Endocrinol. 2013;2013: 587140.

19 Arase Y, Suzuki F, Suzuki Y, Akuta N, Kobayashi M, Kawamura Y, et al. Losartan reduces the onset of type 2 diabetes in hypertensive Japanese patients with chronic hepatitis C. J Med Virol. 2009 Sep;81(9):1584-90.

20 Chukwunonso Obi B, Chinwuba Okoye T, Okpashi VE, Nonye Igwe C, Olisah Alumanah E. Comparative Study of the Antioxidant Effects of Metformin, Glibenclamide, and Repaglinide in Alloxan-Induced Diabetic Rats. J Diabetes Res. 2016;2016:1635361.

21 Ahmadi R, Pishghadam S, Mollaamine F, Zand Monfared MR. Comparing the effects of ginger and glibenclamide on dihydroxybenzoic metabolites produced in STZ-induced diabetic rats. Int J Endocrinol Metab. 2013 Oct;11(4):e10266.

22 Basha RH, Sankaranarayanan C. $\beta$-Caryophyllene, a natural sesquiterpene lactone attenuates hyperglycemia mediated oxidative and inflammatory stress in experimental diabetic rats. Chem Biol Interact. 2016 Feb;245: 50-8.

23 Koren S, Shemesh-Bar L, Tirosh A, Peleg RK, Berman S, Hamad RA, et al. The effect of sitagliptin versus glibenclamide on arterial stiffness, blood pressure, lipids, and inflamma- tion in type 2 diabetes mellitus patients. Diabetes Technol Ther. 2012 Jul;14(7):561-7.

24 Jia J, Xiao Y, Wang W, Qing L, Xu Y, Song H, et al. Differential mechanisms underlying neuroprotection of hydrogen sulfide donors against oxidative stress. Neurochem Int. 2013 Jun;62(8):1072-8.

25 Pandarekandy ST, Sreejesh PG, Harikumaran Thampi BS, Sreekumaran E. Hypoglycaemic effect of glibenclamide: a critical study on the basis of creatinine and lipid peroxidation status of streptozotocin-induced diabetic rat. Indian J Pharm Sci. 2017;79(5):768-77.

26 Chatuphonprasert W, Lao-Ong T, Jarukamjorn K. Improvement of superoxide dismutase and catalase in streptozotocin-nicotinamide-induced type 2 -diabetes in mice by berberine and glibenclamide. Pharm Biol. 2013 Nov;52(4):419-27.

27 Akbar DH, Hagras MM, Amin HA, Khorshid OA. Comparison between the effect of glibenclamide and captopril on experimentally induced diabetic nephropathy in rats. J Renin Angiotensin Aldosterone Syst. 2013 Jun; 14(2):103-15.

28 Williams S, Abbott D, Morfis L, Manwaring P, Diamond T, Howes LG. Effects of glibenclamide on blood pressure and cardiovascular responsiveness in non-insulin dependent diabetes mellitus. J Hypertens. 1998 May;16(5): $705-11$

29 Tada H, Egashira K, Yamamoto M, Ueno H, Takemoto M, Shimokawa H, et al. Glibenclamide, a specific inhibitor of ATP-sensitive $\mathrm{K}+$ channels, inhibits coronary vasodilation induced by angiotensin II-receptor antagonists. J Cardiovasc Pharmacol. 1997 Sep; 30(3):313-9.

30 Martin DE, DeCherney GS, Ilson BE, Jones BA, Boike SC, Freed MI, et al. Eprosartan, an angiotensin II receptor antagonist, does not affect the pharmacodynamics of glyburide in patients with type II diabetes mellitus. J Clin Pharmacol. 1997 Feb;37(2):155-9. 